\title{
Computational prediction of Pho regulons in cyanobacteria Zhengchang $\mathrm{Su}^{1}$, Victor Olman² and Ying $\mathrm{Xu}^{* 2}$
}

Address: ${ }^{1}$ Bioinformatics Research Center and Department of Computer Science, the University of North Carolina at Charlotte, Charlotte, NC 28233, USA and ${ }^{2}$ Computational Systems Biology Laboratory, Department of Biochemistry and Molecular Biology, University of Georgia, Athens, Georgia, USA

Email: Zhengchang Su - zcsu@uncc.edu; Victor Olman - olman@csbl.bmb.uga.edu; Ying Xu* - xyn@bmb.uga.edu

* Corresponding author

Published: 8 June 2007

BMC Genomics 2007, 8:156 doi:10.1/86/147|-2164-8-156
Received: 21 November 2006

Accepted: 8 June 2007

This article is available from: http://www.biomedcentral.com/I47I-2/64/8/156

(C) 2007 Su et al; licensee BioMed Central Ltd.

This is an Open Access article distributed under the terms of the Creative Commons Attribution License (http://creativecommons.org/licenses/by/2.0), which permits unrestricted use, distribution, and reproduction in any medium, provided the original work is properly cited.

\begin{abstract}
Background: Phosphorus is an essential element for all life forms. However, it is limiting in most ecological environments where cyanobacteria inhabit. Elucidation of the phosphorus assimilation pathways in cyanobacteria will further our understanding of the physiology and ecology of this important group of microorganisms. However, a systematic study of the Pho regulon, the core of the phosphorus assimilation pathway in a cyanobacterium, is hitherto lacking.

Results: We have predicted and analyzed the Pho regulons in 19 sequenced cyanobacterial genomes using a highly effective scanning algorithm that we have previously developed. Our results show that different cyanobacterial species/ecotypes may encode diverse sets of genes responsible for the utilization of various sources of phosphorus, ranging from inorganic phosphate, phosphodiester, to phosphonates. Unlike in E. coli, some cyanobacterial genes that are directly involved in phosphorus assimilation seem to not be under the regulation of the regulator SphR (orthologue of PhoB in E coli.) in some species/ecotypes. On the other hand, SphR binding sites are found for genes known to play important roles in other biological processes. These genes might serve as bridging points to coordinate the phosphorus assimilation and other biological processes. More interestingly, in three cyanobacterial genomes where no sphR gene is encoded, our results show that there is virtually no functional SphR binding site, suggesting that transcription regulators probably play an important role in retaining their binding sites.

Conclusion: The Pho regulons in cyanobacteria are highly diversified to accommodate to their respective living environments. The phosphorus assimilation pathways in cyanobacteria are probably tightly coupled to a number of other important biological processes. The loss of a regulator may lead to the rapid loss of its binding sites in a genome.
\end{abstract}

\section{Background}

Cyanobacteria are among the oldest life form on Earth. These organisms inhabit a broad range of ecological environments from fresh water, soil to diverse open oceanographic areas [1]. It is estimated that several cyanobacteria living in the open oceans contribute a significant fraction of Earth's primary production [2]. These bacteria also play important roles in the global cycling of nitrogen and phosphorus $[3,4]$. Therefore their activities have significant impacts on global environmental changes.

Phosphorus is one of the essential elements for all life forms, since it is required for the biosyntheses of nucleotides and phospholipids and for the functional regula- 
tion of proteins through phosphorylation. However, inorganic phosphate $\left(\mathrm{P}_{\mathrm{i}}\right)$, the only form of phosphorus that can be directly utilized by cells, is limiting in most ecosystems [5-7]. Thus, bacteria have evolved to develop various mechanisms to assimilate different phosphoruscontaining compounds available to them. Phosphorus assimilation pathways have been relatively well studied in E. coli $[8]$, and B. subtilis $[9,10]$. For instance, it is generally known that phosphorus assimilation related genes in $E$. coli are regulated by a two-component system comprising the sensor protein, histidine kinase PhoR, and the transcription factor PhoB [8]. PhoR is activated through autophosphorylation when the $\mathrm{P}_{\mathrm{i}}$ level in the environment is low. Activated PhoR then phosphorylates PhoB, thereby activating the latter. Phosphorylated PhoB can either activate or suppress the transcription of genes involved in phosphorus assimilation, resulting in changes in gene expression pattern that accommodate to the availability and the types of phosphorus-containing compounds present in the environment [8]. All the genes whose transcription is regulated by $\mathrm{PhoB}$ in a bacterium are collectively called a Pho regulon. The known members of the Pho regulon in these relatively well-studied species include genes encoding (a) the two-component system such as PhoB and PhoR in E. coli [8] or PhoP and PhoRin B. subtilis $[9,10]$, (b) porins and transporters for transporting phosphorus-containing compounds into the cell, and (c) metabolic enzymes that are used to break down phosphorus-containing compounds to release $\mathrm{P}_{\mathrm{i}}$. In $E$ coli, PhoB is known to bind to at least two tandem direct repeats (Pho boxes) of 8 bp with consensus sequence CTGTACTA separated by an A/T rich 3 bp linker, located 10 bp upstream of the $-10 \sigma^{70}$ binding consensus $\mathrm{TAN}_{3} \mathrm{~T} / \mathrm{A}$ [8]. At least 8 operons comprising 39 genes in E. coli have been identified to be regulated by PhoB [8].

The Pho regulon in cyanobacteria has only been relatively well studied in Synechocystis PCC 6803 (PCC6803) $[11,12]$. Previous studies have shown that a two-component system comprising the sensor kinase SphS (orthologue of PhoR in E. coli) and the response regulator SphR (orthologue of PhoB in E. coli) works in a similar manner to sense the $\mathrm{P}_{\mathrm{i}}$ level in the environment and to regulate the expression of genes that are directly involved in phosphorus assimilation, forming a Pho regulon similar to that in E coli $[11,12]$. However, only three operons have been identified to be activated by the phosphorylated SphR, including the operons sphX-pstS1-C1-A1-B1-B1' and pstS2$C 2-A 2-B 2$, each of which encodes an ABC type high-affinity $\mathrm{P}_{\mathrm{i}}$ uptake system, and the operon $p h o A-n u c H$, where phoA encodes an alkaline phosphatase, and nucA an extracellular nuclease [12]. The singleton operon urtA that encodes a subunit of urea transporter is repressed under phosphorus limitation by an unknown mechanism [12]. SphR activates the transcription of genes by binding to at least three tandem direct repeats (Pho boxes) of $8 \mathrm{bp}$, but with a consensus sequence different than that in $E$. coli, namely, CTTAACCT, in the promoter regions of these genes [12]. It is unknown whether the Pho regulon in PCC6803 includes any other genes in addition to these three operons. Furthermore, although $s p h R$ is encoded in 15 other sequenced cyanobacterial genomes besides PCC6803, little is known about which genes in these genomes are regulated by SphR. In this work, we have predicted the Pho regulons in 16 sequenced cynaobacterial genomes that encode the $s p h R$ gene using an efficient phylogenetic footprinting based motif scanning algorithm [13]. Interestingly, three cyanobacterial genomes, i.e., Prochlorococcus marinus CCMP1375 (CCMP1375) and Synechococcus sp. CC9311 (CC9311) and Synechococcus sp. CC9902 (CC9902) that do not encode the sphR gene, provide us a rare and highly valuable opportunity to examine the turnover of the cis-regulatory binding sites after their regulators were lost during the course of evolution.

\section{Results}

\section{Conservation of the DNA binding domain of SphR}

We have identified orthologues of SphR of PCC6803 (sll0337) in 16 out of the 19 sequenced cyanobacterial genomes using the criterion described in the Methods section. Interestingly, the genomes of CCMP1375, CC9311 and CC9902 do not encode the sphR gene. As shown in Figure 1A, the DNA binding domain of SphR of the 16 cyanobacteria is highly conserved, suggesting that the DNA binding sites of SphRs in these species are likely to be conserved. In particular, the amino acid residues of PhoB of E. coli that are in direct contact with the Pho boxes (marked by red dots) as revealed by the crystal (tertiary) structure of the PhoB-DNA complex [14] are also highly conserved in the SphR sequences (Figure 1A). This may explain why both PhoB of E. coli and SphR of PCC6803 bind to $8 \mathrm{bp}$ tandem repeats with a 3 bp linker $[8,12]$. We therefore infer that all the SphRs in these cyanobacterial genomes bind to cis-regulatory sites with a similar sequence structure. The phylogenetic relationship among these SphR sequences is shown in Figure 1B.

\section{Profiles of the SphR binding sites predicted by phylogenetic footprinting}

When the phylogenetic footprinting technique is applied to the pooled 50 upstream regions (data not shown) of the operons whose genes are orthologous to those that are regulated by SphR in PCC6803, highly conserved two tandem repeats with a linker are identified in 30 these sequences by our motif finding program CUBIC [15] with a cutoff score at $p<0.001$. We assume that these motifs are putative Pho boxes, and are shown in Table 1. The three known SphR binding sites in PCC6803 are accurately recovered (Table 1 ), suggesting that most of the identified motifs are likely to be true Pho boxes. Shown in Figure 1C 

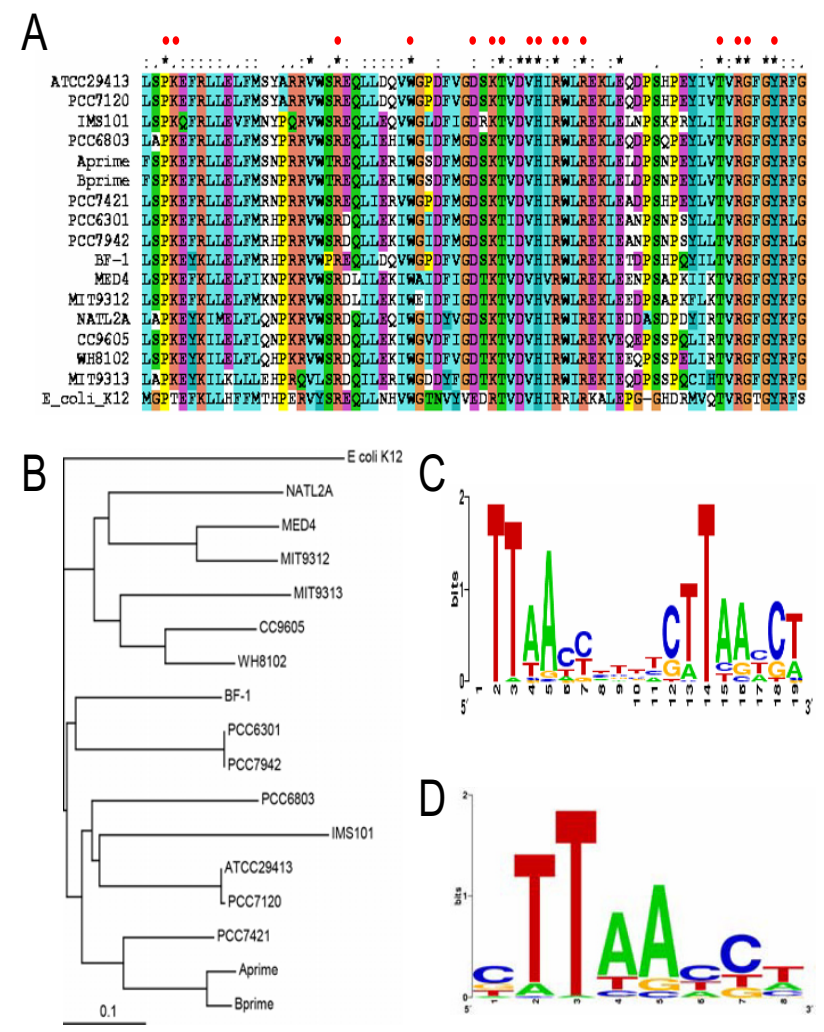

\section{Figure I}

Profiles of the Pho boxes constructed by phylogenetic footprinting. A. Multiple sequence alignments of the DNA binding domains of the SphR sequences of 16 sequenced cyanobacteria and that of the PhoB of $E$. coli, generated by the ClustalX program. The red dots show the positions in PhoB sequence of $E$. coli that are in direct contact with the Pho boxes of the DNA sequence in the crystal structure of PhoB-DNA complex. B. Phylogenetic relationships of SphR of the 16 genomes in our analyses. The tree was generated using the neighborjoining method based on the multiple sequence alignments by the ClustalX program using the default settings, and is rooted at $\mathrm{PhoB}$ of $E$. coli K I2. Scale bar, substitutions per position. C. Logo representation of the profile of the putative two tandem repeats of Pho boxes plus the linker found by phylogenetic footprinting. D. Logo representation of the profile of the combined putative Pho boxes of the two tandem repeats shown in panel (C). Logos were generated by the Weblogo server [32].

is the logo representation of the sequence profile of these putative two tandem Pho boxes plus the linker; Figure 1D is the logo representation of the sequence profile of the combined putative Pho boxes of the two tandem repeats shown in 1C. However, such conserved two tandem repeats are not found for any orthologous genes from CC9605 MIT9313, CC9311 and CC9902, suggesting that these genes do not bear Pho boxes or their Pho boxes differ largely from the conserved ones, which cannot be iden- tified by CUBIC. Interestingly, CUBIC finds high scoring tandem Pho boxes for the pstC-A-B operon in CCMP1375, although this genome does not encode the sphR gene, thus they are unlikely to be functional.

\section{Genome wide prediction of SphR binding sites in each genome}

We have predicted all possible SphR binding sites in each of the 19 sequenced genomes by scanning their genome sequences with the two profiles of the Pho boxes that we have constructed above (shown in Figure 1C and 1D), assuming that a SphR binding site consists of three Pho boxes with two $3 \mathrm{pb}$ linkers. Given profiles of multiple cooccurring binding sites, our binding-site scanning algorithm computes a log-odds ratio function $\operatorname{LOR}(s)$ of the scores $(s)$ of the candidate sites in a genome. LOR is the ratio of the probability of finding a SphR binding site in the intergenic regions divided by the probability of finding a SphR binding sites in randomly chosen coding regions. We use coding regions as control sequence because it is less likely that functional SphR binding sites would occur in coding regions (for details see Methods). We have argued that our scoring function captures the essence of true cis-binding sites in a genome so that if the genome contains functional binding sites described by the profiles, its $\operatorname{LOR}(s)$ will become positive when the score value $s$ increases beyond some threshold; and the larger a $\operatorname{LOR}(s)$ value is, the higher the confidence for the prediction is [13]. In contrast, if a genome only contains similar sequences occurring by chance, then its $\operatorname{LOR}(s)$ will be around 0 or lower independent of the increase of the score value $s$ [13]. As shown in Figure 2, when $s$ increases beyond a certain value, the $\operatorname{LOR}(s)$ becomes positive for the 16 genomes that encode the sphR gene, and the $L O R(s)$ values are generally high, suggesting that these 16 genomes contain more high scoring SphR binding sites than one would expect by chance, thus these high scoring sites are likely to be functional. In contrast, the $\operatorname{LOR}(s)$ for the genomes of CCMP1375, CC9311 and CC9902, which do not encode the sphR gene, becomes negative when $s$ increases beyond a certain value, indicating that the putative SphR binding sites found in the intergenic regions of these genomes are likely to occur by chance. Therefore they are unlikely to be functional. We argue that the lack of strong signals for possible SphR binding sites in CCMP1375, CC9311 and CC9902 is unlikely caused the lack of representative Pho boxes from these genomes in the profiles used in our scanning algorithm. Our profiles do not contain any representative sites from CC9605 and MIT9313 (Table 1), yet their LOR(s)' are still positive. In contrast, though CCMP1375 has representative sites in our profiles (Table 1), its LOR(s) is negative. We conclude that our algorithm is not biased towards the genomes from which the profiles are constructed and it is robust enough to uncover true SphR binding sites in closely 
Table I: Genes with two tandem Pho boxes found in their promoter regions by phylogenetic footprinting

\begin{tabular}{|c|c|c|c|c|c|c|}
\hline Genome & Rank' & Tanscription unit & Names & Pho Boxes ${ }^{2}$ & Positon ${ }^{3}$ & Score ${ }^{4}$ \\
\hline \multirow[t]{6}{*}{ ATCC294I3 } & 7 & Ava_254I & phoA & GTTAATCTtcaGTTAACCTaaaTTTATATT & -111 & 9.07 \\
\hline & 11 & Ava_45I6 Ava_45I5 Ava_45I4 Ava_45I3 & pstS2 pstC2 pstA2 pstB2 & TATTTGTAtatTTTAACTTgaaATTAACCA & -403 & 8.75 \\
\hline & & Ava_45/2 & 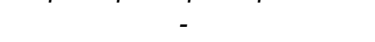 & & & \\
\hline & 17 & Ava_2477 & pstSI pstCI pstAl, pstBI' & TTTTTCCT tgtTTTAACCTttcGTTAACCT & -66 & 8.54 \\
\hline & 27 & Ava_37/6 & $\operatorname{sph} X$ & CTTTACTGataCATCAACTtttTTTGATGA & -104 & 8.29 \\
\hline & 47 & Ava_4728 & nucH & TTTTACCTtttCCTATTATtctCTTAATCT & -174 & 8.04 \\
\hline \multirow[t]{2}{*}{ Aprime } & 3 & CYA_1552 CYA_1553 CYA_1554 CYA_1555 & pstS pstC pstA pstB & AATAACCTgaaTTTAACCTcttGTTAACCA & -175 & 10.11 \\
\hline & & CYA_1556 CYA_I557 & -- & & & \\
\hline Bprime & 10 & CYB_2765 CYB_2766 & nucH - & CTTAAGCGtacCTTAACTGcccCTTTACTC & -91 & 9.49 \\
\hline BF-I & 2 & $\operatorname{tr} 2164 \operatorname{tr} 2165$ trr2166 t/r2167 & sphX pstC pstA pstB & TTTAAACAaacTTTTACCTtctCTTAACTT & -23 & 9.91 \\
\hline IMSIOI & 21 & Tery_3534 & pstS & TTTGATATtttTTTAACCTgttCTTAATCT & -164 & 8.18 \\
\hline \multirow[t]{3}{*}{ PCC630I } & 1 & syc0545_d & nucH & TTTAAAGTgctGTTAATCCttcCTTTACCG & -276 & 9.76 \\
\hline & 2 & $\begin{array}{c}\text { sycl 66I_d sycl 662_d sycl 663_d sycl 664_d } \\
\text { sycl 665_d sycl 666_d }\end{array}$ & $\begin{array}{l}s p h X \text { pstS pstC pstA } \\
\text { pstB pnp }\end{array}$ & CTTAGGGTcgcCTTAATAGgctGTTAAACT & -48 & 9.75 \\
\hline & 4 & syc0163_d & phoA & TTTAACTAtttCATAATCTattCTCAATCT & -216 & 9.41 \\
\hline \multirow[t]{4}{*}{ PCC6803 } & I & sll0679 & $\operatorname{sph} X$ & TTTAACCAaacCTTTACTAgggCTTAACCT & -105 & 10.31 \\
\hline & 2 & $s / r / 247$ slr $/ 248$ slr $/ 249$ slr 1250 & pstS2 pstC2 pstA2 pstB2 & CTTAATTCtatCTTAATTTс gaCTTAATCA & -303 & 10.10 \\
\hline & 7 & sll0654 sll0656 & phoA nucH & CTTAACCTtttCATAGTCT aacCATAAGTT & -156 & 8.80 \\
\hline & 14 & sll0680 sll068I sll0682 sll0683 sll0684 & pstSI pstCI pstAI pstBI pstBI' & ATTCCATAgacCTTAACCTtccCTTTACCA & -73 & 8.46 \\
\hline \multirow[t]{4}{*}{ PCC7I20 } & 6 & alr5291 & PhoA & ATTAATCTttaGTTAACCTgaaTTTATATT & -223 & 9.25 \\
\hline & 10 & all4575 all4574 all4573 all4572 & pstSI pstCI pstAI pstBI & TTTTСТСТ tgaTTTAACCTttcGTTAACCT & -64 & 8.97 \\
\hline & 38 & alr 1094 alr 1095 & $\operatorname{sph} X$ gap3 & СTTTACTAataCATCAACTtttATTGATGA & -106 & 8.19 \\
\hline & 51 & alr0276 & nucH & TTTTACCTtctCCTATTATtctCTTAATCT & -178 & 8.05 \\
\hline PCC742I & 31 & glr0445 glr0446 glr0447 glr0448 & pstS pstC pstA pstB & GTTAACCTtgcCTTAATCGaatTTCGTTAC & -80 & 8.64 \\
\hline \multirow[t]{4}{*}{ PCC7942 } & 1 & Synpcc7942_1000 & nucH & TTTAAAGTgctGTTAATCCttcCTTTACCG & -310 & 9.72 \\
\hline & 2 & Synpcc7942_2445 & $\operatorname{sph} X$ & CTTAGGGTcgcCTTAATAGgctGTTAAACT & -46 & 9.71 \\
\hline & 4 & Synpcc7942_1392 & phoA & TTTAACTAtttCATAATCTattCTCAATCT & -214 & 9.37 \\
\hline & & Synpcc7942_244I & - & & & \\
\hline CCMPI375 & 18 & Pro0598 Pro0597 Pro0596 & pstC pstA pstB & CTTAACTCtctCTTAAACAtgcACTTGATA & -47 & 7.36 \\
\hline \multirow[t]{2}{*}{ MED4 } & 14 & PMM07IO & pstS & TTTAACTAgccCTTAATCAtttCTTATATT & -245 & 8.07 \\
\hline & 24 & PMM0723 PMM0724 PMM0725 & pstC pstA pstB & TTTATATAtacCTTTAACCcttCTTAAAGT & -48 & 7.67 \\
\hline MIT93। 2 & 8 & PMT93/2_0722 & pstS & TTTAAAGAgaaCTTAAACTtccCTTAAATT & -231 & 8.49 \\
\hline \multirow[t]{2}{*}{ NATL2A } & 13 & PMN2A_03II PMN2A_03I0 PMN2A_0309 & pstC pstA pstB & ATTAACCCtctCTTAGTGAaaaATTTGAAA & -47 & 7.52 \\
\hline & & PMN2A_0308 PMN2A_0307 & -- & & & \\
\hline WH8I02 & 1 & SYNW239I SYNW2390 & - phoA & TTTGATCAgatCTTAAACTattCCTAACTT & -59 & 11.18 \\
\hline
\end{tabular}

I. The rank of three tandem Pho boxes recovered by our scanning algorithm.

2. Bold, the two tandem Pho boxes identified by phylogenetic footprinting; light, the additional Pho box found by our scanning algorithm; Italics, known Pho boxes.

3. Positions of the Pho boxes relative to the first codon in the operon.

4. The scores of the three tandem Pho boxes recovered by our scanning algorithm. 
related cyanobacteria even when no representative site from them is included in the profiles. In addition, the results show that our scanning algorithm can tolerate some level of noise caused by including non-functional sites in the profiles. Hence, we conclude that CCMP1375, CC9311 and CC9902 are unlikely to contain functional SphR binding sites represented by the profiles. On the other hand, we have predicted putative members of the Pho regulons in the other 16 genomes based on the presence of a putative SphR binding site found in their promoter regions at a statistical significance level of $p<0.01$ (see Methods). These results are listed in the Additional file 1: Table 1s-16s. The most interesting observations from these predictions are summarized below.
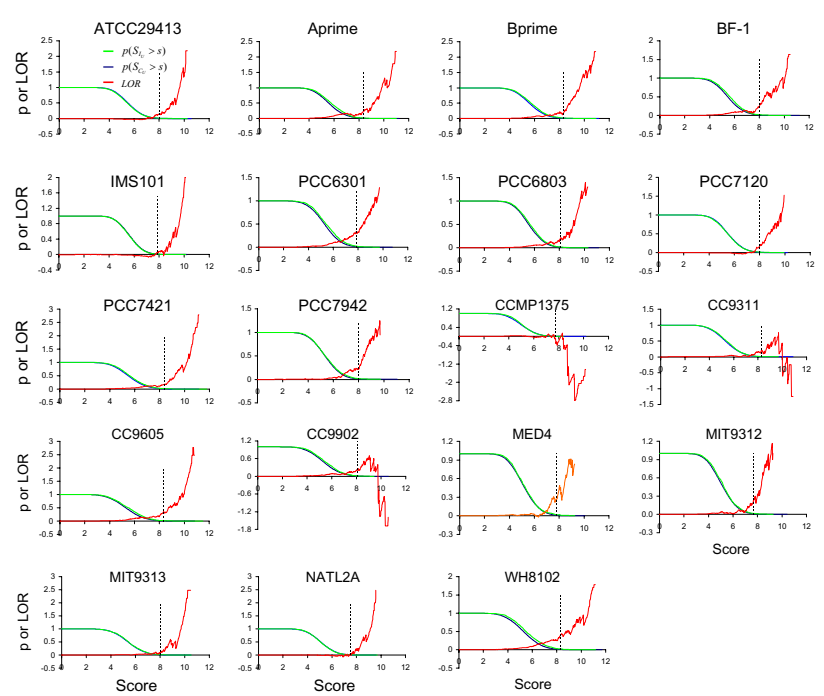

\section{Figure 2}

Genome wide prediction of Pho regulons in the 19 sequenced cyanobacterial genomes. Green lines represent the probability $\left(p\left(S_{I_{U}}>s\right)\right)$ that putative three tandem Pho boxes found in the promoter region $I_{U\left(g_{1}, \ldots, g_{n}\right)}$ of an operon $U\left(g_{1}, \ldots, g_{n}\right)$ have a score greater than $s(s$ is a positive number); blue lines represent the probability $\left(p\left(S_{C_{U}}>s\right)\right.$ ) that putative three tandem Pho boxes found in a randomly chosen coding region $C_{U\left(g_{1}, \ldots, g_{n}\right)}$ with the same length as $I_{U\left(g_{1}, \ldots, g_{n}\right)}$ have a score greater than $s$ ( $s$ is a positive number); Red lines represent the log-odds ratio function defined as $\operatorname{LOR}(s)=\ln \left(p\left(S_{I_{U}}>s\right) / p\left(S_{C_{U}}>s\right)\right)$. The doted vertical line in each panel shows the value of $s$, such that $p\left(S_{C_{U}}>s\right)<0.01$, and $s$ is the cutoff for SphR binding site predictions. $p\left(S_{C_{U}}>s\right)$ is also used to estimate the $p$ value.

\section{Pho regulon members that are directly involved in phosphorus assimilation process}

\section{I Two-component system sphS/sphR genes}

The phosphorus sensor kinase sphS and response regulator $s p h R$ genes are predicted to be in the same operon in ATCC29413, PCC6301, PCC7120, PCC7942, CC9605, MED4, MIT9312, NATL2A and WH8102, but they are clearly split into separate operons in Aprime, Bprime, BF1, PCC6803 and PCC7421 (Figure 3). In addition, the

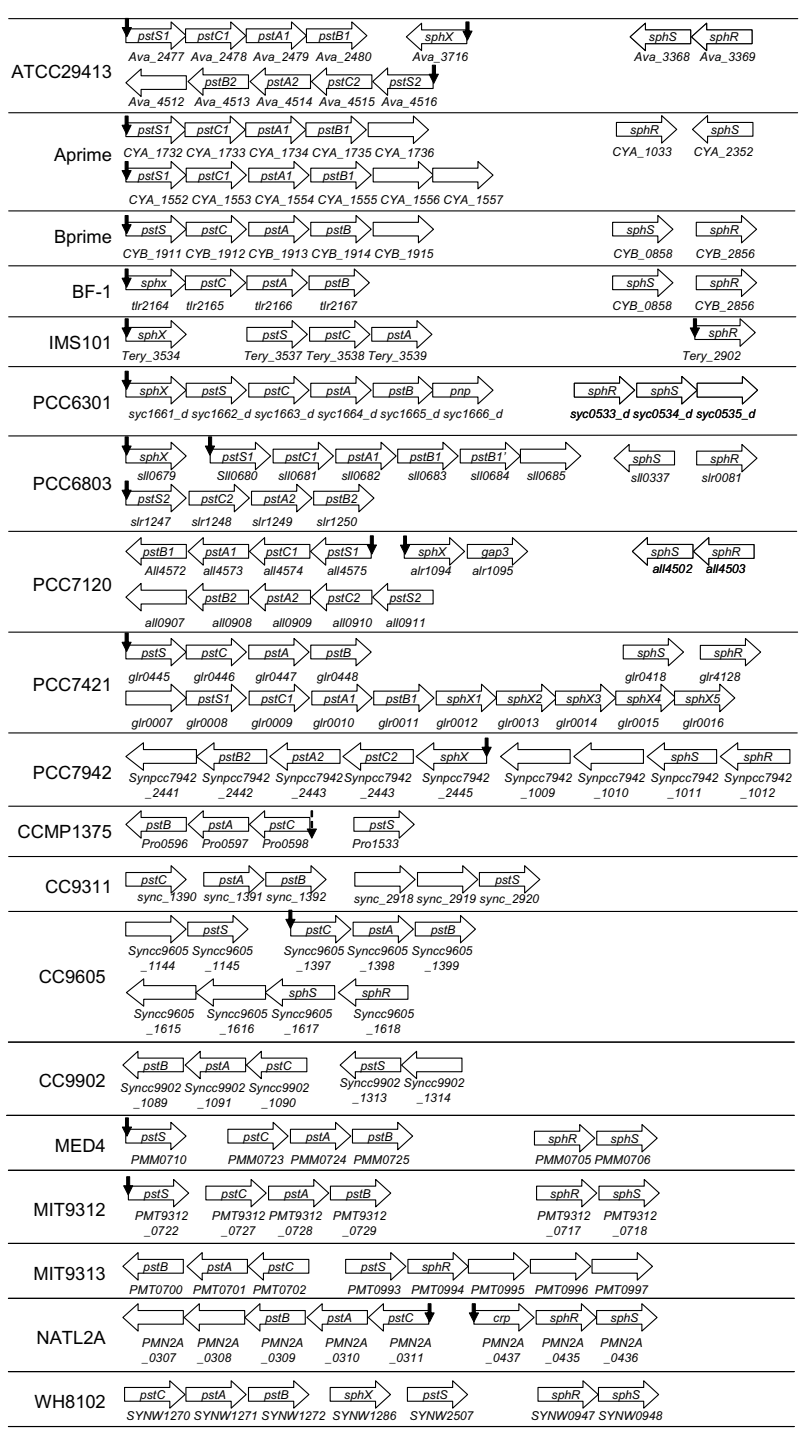

\section{Figure 3}

Genomic organization of the two-component system sphS/ sphR genes and the $P_{i}$ uptake system pst genes in cyanobacteria. A vertical arrow indicates that three tandem Pho boxes are found for the predicted operon with $p<0.01$. A doted arrow represents a non-functional SphR binding site. The order of the operons does not reflect their actual positions on the chromosome, but rather it is a schematic illustration of the operons. 
sphS gene is not encoded in IMS101; and the gene in MIT9313 is frame-shifted [16,17], and therefore is unlikely to be functional. Thus, SphS in IMS101 and MIT9313 may be activated by other regulators as suggested for MIT9313 where multiple regulatory genes are induced by P starvation [17]. High scoring tandem Pho boxes are found for the singleton operon sphR in IMS101 (Tery_2902, Table 5s and Figure 3) and for the operon crpsphR-sphS in NATL2A (PMN2A_0435-0437, Table 15s and Figure 3 ), suggesting that these operons are regulated by SphR in their respective genomes. However, the sphs/sphR genes in the other cyanobacterial genomes are unlikely to be regulated by SphR. Thus the auto-regulation of the phoBR operon found in E. coli is not a general rule.

\subsection{High affinity $A B C$-type $P_{i}$ pst transporter complex genes}

All the 19 genomes analyzed in this study encode at least one Pst transporter complex. In most of these genomes, the complex is composed of the phosphate binding subunit PstS, the transmembrane subunits PstC and PstA, and the ATP-binding subunit PstB, whose genes form an operon in the form of $p s t S-C-A-B$. However, the $p s t S$ gene is split out to form a singleton operon in CCMP1375, CC9905, MED4, MIT9312, and MIT9313 (Figure 3). Furthermore, in the genomes of ATCC29413, IMS101, PCC6301, PCC6803, PCC7421, PCC7120, PCC7942, BF1 and WH8102, the Pst transporter complex includes an additional phosphate binding subunit SphX. The sphX gene in BF-1, PCC6301 and PCC7942 forms an operon with the genes of the other subunits of the complex in the form of sphX-pstS-C-A-B (Figure 3). Interestingly, multiple copies of the sphX gene are found in downstream of one of the two pst operons in PCC7421 (Figure 3). High scoring tandem Pho boxes are found for most operons that encode one or more pst genes (Figure 3), except for those in the genomes CC9902, CC9311, MIT9313 and WH8102, where no Pho box is found for the operon pstC$A-B$ and the pstS gene. As mentioned before, Pho boxes are found for the pstC-A-B operon in CCMP1375 through phylogenetic footprinting, but they are unlikely to be functional due to the lack of $s p h R$ gene in the genome. The reason why these Pho boxes remain after $s p h R$ was lost is unknown. On the other hand, while we did not find any tandem Pho boxes for the pstC-A-B operon in CC9905 using phylogenetic footprinting (Table 1), our scanning algorithm identifies a putative SphR binding site in its promoter region with a score value only marginally above the cutoff (Table 11s). The absence of SphR binding sites for the pstS gene and the pstC-A-B operon in CC9311 and CC9902 might be due to the missing of the sphR gene in their genomes as discussed below. The lack of SphR binding sites for the $p s t C-A-B$ operon and the pstS gene in MIT9313 and WH8102 might be due to the relative abundance of phosphate in their environments as evidenced by the loss of the sphS gene in the MIT9313 genome. In addi- tion, while experiments have shown that the gene cluster sphX-pstS1-C1-A1-B1-B1' in PCC6803 form an operon that is regulated by SphR through the three tandem Pho boxes in the upstream of the sphX gene [12], we predicted that $s p h X$ and pstS1-C1-A1-B1-B1' can form sub-transcriptional units, and a high scoring SphR binding site is found in the upstream intergenic region of the pstS1 gene. The possible function of this putative SphR binding site warrants further experimental investigation.

\subsection{Phosphonate transporter complex and C-P lyase genes}

Orthologous genes of the phosphonate transporter complex and the C-P lyase in E. coli are found in the genomes of PCC7120, IMS101 and Bprime, suggesting that these species are capable of utilizing phosphonates as a phosphorus source for growth. The importance of phosphonates for the prevalence of IMS101 in the vast open oceans has recently been experimentally demonstrated [4]. Although in E. coli, genes encoding the phosphonate transporter system (phnCDE) and those encoding the C-P lyase complex (phnFGHIJKLMNOP) are located in the same operon in the form of phnC-D-E-F-G-H-I-J-K-L-M-N$O-P$ and are regulated by PhoB $[18,19]$, the phn genes are split into two operons in Bprime and PCC7120, and the phnCDE genes are duplicated in IMS102 (Figure 4). High scoring SphR binding sites are found for the predicted operons phnC-D-E-G-H-I-J-K-L-M in Bprime, phnD-C-EE2-G-H-I-J-K-L-M in IMS101 and phnC-D-all229-E in PCC7120, suggesting that they are likely to be regulated by SphR. These results are consistent with the findings in IMS101 that when tested, the phnD and phnJ genes were activated by a phosphorus-deficient medium [4] in which SphR was presumably activated by an unknown regulator.

\subsection{Phosphatase genes}

Most of the 19 cyanobacterial genomes encode alkaline phosphatase genes, possibly of different families. The

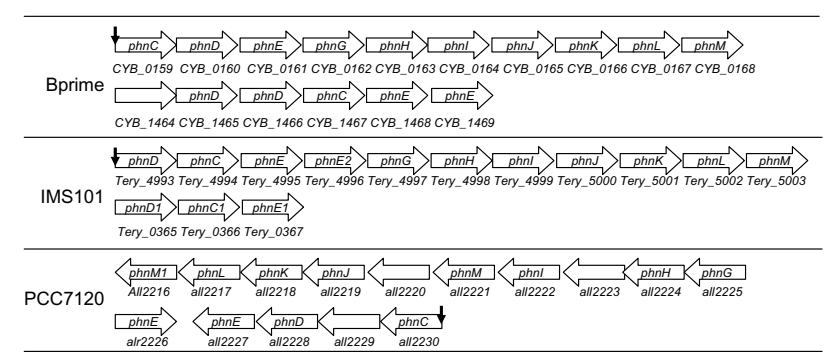

\section{Figure 4}

Genomic organization of the phn genes in cyanobacteria. A vertical arrow indicates that three tandem Pho boxes are found for the predicted operon with $p<0.01$. The order of the operons does not reflect their actual positions on the chromosome, but rather it is a schematic illustration of the operons. 
PhoA family members are found in ATCC29413 (Ava_2541), Aprime (CYA_1059), Bprime (CYB_0274), MED4 (PMM0708), MIT9312 (PMT9312_0720), NATL2A (PMN2A_0439), PCC6301 (syc0163_d), PCC6803 (sll0654), PCC7120 (alr5291), PCC7421 (gll0893), PCC7942 (Syncc7942_1392), and WH8102 (SYNW2390 and SYNW2391), mostly as a singleton operon except for Aprime, Bprime, MED4, NATL2A, PCC6803 and WH8102, in which the phoA gene is predicted to form an operon with other genes (Figure 5). High scoring SphR binding sites are found in their promoter regions except for CYA_1059 in Aprime, CYB_0274 in Bprime and PMT9312_0720 in MIT9312 (Figure 5). Furthermore, high scoring SphR binding sites are found for the PhoD family members encoded in PCC7120 (alr4976 and alr2234) and PCC7421 (gll0490), and for other families of alkaline phosphatases encoded in Bprime (CYB_1198) and Aprime (CYA_0781) (Table 5). These results suggest that as in E. coli, alkaline phosphatases in cyanobacteria play an important role in utilizing the $\mathrm{P}_{\mathrm{i}}$ moiety in various organic compounds, and this process is probably highly regulated by SphR in most species/ecotypes.

Aprime

\section{Figure 5}

Genomic organization of alkaline phophosphatases and extracellular nuclease nucH genes in cyanobacteria. A vertical arrow indicates that three tandem Pho boxes are found for the predicted operon with $p<0.01$. The order of the operons does not reflect their actual positions on the chromosome, but rather it is a schematic illustration of the operons. The label alk represents an alkaline phosphatase of an uncharacterized type.

\subsection{Extracellular nuclease nucH gene}

It has been shown that the phoA-nucH operon in PCC6803 is activated under P limitation, presumably through binding of phosphorylated SphR to the four tandem Pho boxes in its promoter region [12], suggesting that $\mathrm{NucH}$ might play a crucial role in the utilization of the $\mathrm{P}_{\mathrm{i}}$ moiety in the nucleic acids in its environment. However, the nucH gene is not found in the genomes of BF-1, CCMP1375, MED4, MIT9312, MIT9313, NATL2A CC9602, CC9605, WH8102, which might reflect the fact that the presence of nucleic acids in their ecological niche is less likely, such as in the oligotrophic open oceans. In the genomes where the $n u c H$ gene (Figure 5) is encoded, it is predicted to form a singleton operon except for PCC6803, PCC7421 and Bprime. High scoring SphR binding sites are found for the $n u c H$ genes in the genomes of ATTCC29413 (Ava_2698, Table 1s, and Figure 5), PCC6301 (syc0545_d, Table 6s), PCC7120 (alr0276, Table 8s) and PCC7942 (Synpcc7942_1000, Table 10s, Figure 5), suggesting that they are under the regulation of SphR in these species/ecotypes.

\section{Putative Pho regulon members likely to be involved in phosphorus assimilation related global responses}

It is interesting that high scoring SphR binding sites are found for numerous genes that might not be directly involved in the phosphorus assimilation pathway but rather in some other important biological processes, such as photosynthesis, carbon fixation and nitrogen assimilation (Table 2). We have also predicted that three other classes of genes bear SphR binding sites, namely, transporters/porins, kinases and transcription factors (Table 2). In addition, high scoring SphR binding sites are found for numerous conserved hypothetical genes across all the cyanobacterial genomes that encode the sphR gene, which are listed in Table 1s-16s. Their specific functions deserve further experimental characterization.

\section{Discussion}

\section{Degradation of SphR cis-binding sites after SphR was lost}

The low $\operatorname{LOR}(s)$ values of the putative SphR binding sites in the genomes of CCMP1375, CC9311 and CC9902, in which no $s p h R$ gene is found, indicate that these genomes might not contain functional SphR binding sites. Since all the CCMP1375, CC9311 and CC9902 genomes encode other phosphorus assimilation related genes, such as the pst transporter genes, which are in general regulated by SphR in the other 16 cyanobacterial genomes that encode the sphR gene, it is reasonable to assume that the last common ancestor of the cyanobacteria harbored ancient versions of sphR, sphS and other phosphorus assimilation related genes that were regulated by the SphS/SphR system. Therefore, we conclude that the sphR and sphS genes in CCMP1375, CC9311 and CC9902 were lost during the 
Table 2: Genes bearing a putative SphR binding site with unknown function in $P$ assimilation

\begin{tabular}{|c|c|c|c|c|c|c|}
\hline Genomes & Photosynthesis & Carbon fixation & $\begin{array}{l}\text { Nitrogen } \\
\text { assimilation }\end{array}$ & $\begin{array}{l}\text { Transporters } \\
\text { or porins }\end{array}$ & Kinases & $\begin{array}{l}\text { Transcription } \\
\text { factors }\end{array}$ \\
\hline ATCC294I3 & & & & & $\begin{array}{l}\text { Ava_0064 } \\
\text { Ava_2524 }\end{array}$ & Ava_2324 \\
\hline Aprime & CYA_0229 & CYA_2357 & & & & $\begin{array}{l}\text { CYAI54I } \\
\text { CYA_006I }\end{array}$ \\
\hline Bprime & & & & $\begin{array}{l}\text { CYB_1898 } \\
\text { CYB_2516 }\end{array}$ & CYB_2465 & \\
\hline BF-I & & & & & & $\begin{array}{l}\text { tll1021 } \\
\text { tll I024 } \\
\text { tll I025 }\end{array}$ \\
\hline IMSIOI & & & & $\begin{array}{l}\text { Tery_2377 } \\
\text { Ter_45 I5 }\end{array}$ & Tery422I & Tery_2828 \\
\hline PCC630I & $\begin{array}{c}\text { syc_2044_d-2045_d } \\
\text { syc_1093_c }\end{array}$ & & syn_1338_c & & & $\begin{array}{l}\text { sycl } 747 \text { dd } \\
\text { syc0684_c } \\
\text { syc0685_c }\end{array}$ \\
\hline PCC6803 & $s|r||8|$ & & slr0753 & & & $\begin{array}{l}\text { SIrl I8I } \\
\text { sll I555 }\end{array}$ \\
\hline PCC7I20 & alr0528-0537 & & glr306I & all3/32 & $\begin{array}{l}\text { all0542 } \\
\text { alr I I } 71 \\
\text { alr5272 }\end{array}$ & $\begin{array}{l}\text { alr I I } 70 \\
\text { alr5224 } \\
\text { all0638 } \\
\text { all0637 }\end{array}$ \\
\hline PCC742I & gvip499 & gll3548 & & gll0367 & & \\
\hline PCC7942 & $\begin{array}{l}\text { Synpcc7942_0424 } \\
\text { Synpcc7942_2048 }\end{array}$ & & Synpcc7942_0169 & & Synpcc7942_/356-1357 & $\begin{array}{l}\text { Synpcc7942_2356 } \\
\text { Synpcc7942_1355 }\end{array}$ \\
\hline & -2049 & & & & & \\
\hline CC9605 & $\begin{array}{c}\text { Syncc9605_1756 } \\
\text { Syncc9605_1831 } \\
\text { Syncc9605_0307 } \\
\text { Syncc9605_0442-0444 } \\
\text { Syncc9605_2579 }\end{array}$ & & & & & \\
\hline MED4 & & PMM I 438-I 439 & & & РMM0709 & PMM0572 \\
\hline MIT93I2 & & PMT93 I2_1542-1550 & & & PMT93I2_072I & PMT93 I2_0577 \\
\hline MIT93। 3 & PMTI 046 & & & & & \\
\hline NATL2A & & & & & & PMN2A_0435 \\
\hline WH8I02 & $\begin{array}{c}\text { SYNW0817 } \\
\text { SYNWI999-2200I }\end{array}$ & & & & SYNW0246 & SYNWIOI9 \\
\hline
\end{tabular}

course of evolution. Interestingly, CCMP1375 inhabits a lower layer of the sea water in oligotrophic open oceans [20], and both CC9311 [21] and CC9902 [22] were isolated from costal seawater, both of which are known to be relatively rich in $P_{i}[21,23]$. It is likely that the abundant $P_{i}$ in these niches relieved the selection pressure to retain $s p h R / s p h R$ genes in these organisms, and have resulted in their complete loss. Alternatively, the function of SphR could have been hitchhiked by another regulator, which resulted in its loss in these genomes. In any event, the loss of $s p h R$ would have in turn led to the degradation of otherwise conserved Pho boxes as it has been shown that the conservation of cis-regulatory binding sites is due to the constant purifying selection exerted from the binding interface of their trans-regulators [24].

\section{Phosphorus assimilation pathways and their possible coupling to other biological pathways}

Our analysis results indicate that cyanobacteria as a group of widely distributed microorganisms can utilize a broad range of phosphorus sources, including free $\mathrm{P}_{\mathrm{i}}, \mathrm{P}_{\mathrm{i}}$-containing organic compounds and C-P bond-containing phosphonates, as the relevant transporters and metabolic enzymes are found in their genomes. However, the distribution of these genes in each genome is quite different, reflecting the unique environment where a specific organism inhabits. The only genes that are ubiquitously encoded in all the 19 genomes are those that encode the four subunits of high affinity $P_{i}$ Pst uptake system, namely, the pstS, pstC, pst $A$ and $p s t B$ genes, suggesting that $\mathrm{P}_{\mathrm{i}}$ is probably the most widely available source of phosphorus for cyanobacteria despite its low concentration in some environments. While many genes that are directly involved in the phosphorus assimilation pathway are predicted to be regulated by SphR, some others are not. This is in sharp contrast to their orthologues in E. coli, where all these genes are regulated by PhoB [8]. In addition, the regulatory modes of the phosphorus assimilation genes in cyanobacteria are also highly diversified in terms of the distribution of the predicted SphR binding sites for the 
orthologous genes, reflecting the heterogeneity and complexity of the environments where they inhabit.

Intriguingly, numerous putative new members of the Pho regulon are predicted in each of the analyzed genomes containing the sphR gene. Many of these appear to not be directly associated with phosphorus assimilation but instead encode proteins that are involved in photosynthesis, carbon fixation, nutrients/solutes transportation, signal transduction (sensor kinases), and transcription regulation (response regulators) (Table 2). A SphR binding site in the promoter region of these genes might serve as a mechanism of cross-talk between the phosphorus assimilation pathway and the pathways in which they play a direct role. It has in fact been shown that nitrogen fixation in IMS101 is restricted by phosphorus [3], an indication of coordination between the phosphorus assimilation and the nitrogen fixation. Interestingly, we have predicted several nitrogen assimilation genes to be putative members of the Pho regulon in several species/ ecotypes (Table 2). In addition, it has been shown that in E. coli [25], B. subtilis [26], MED4 [17] and MIT9313 [17], $P$ limitation is correlated with up- or down-regulations of both phosphate assimilation related proteins/genes and other proteins/genes. For instance, in E. coli, only 39 Pho regulon genes have been experimentally shown to be directly involved in phosphorus assimilation, while the number of proteins involved in the global response elicited by $\mathrm{P}$ limitation could be much larger as it has been shown previously that at least 413 genes are involved in the global response to P limitation in E. coli [25]. While some of these 413 genes might be under the direct control of PhoB, the others could be the results of cross-talks between the phosphorus assimilation and other biological pathways. This is probably achieved through a network of signal transduction proteins and transcriptional regulators that are directly or indirectly regulated by PhoB. Such a regulatory network is also likely to exist in cyanobacteria, as it was shown that $\mathrm{P}$ limitation results in changes in expression of 34 and 178 genes in MED4 and MIT9313, respectively [17], though only 5 and 2 of them are predicted to bear putative SphR binding sites, respectively. This suggests that the other genes are probably regulated by other regulators. Alternatively, these genes could be regulated by SphR through different binding motifs. In addition, it has been shown that the expression of the urtA gene in PCC6803 (slr0447) [12] and in MED4 (PMM0970) [17] is strongly repressed during phosphorus starvation. However, no $s p h R$ binding site has been experimentally identified in PCC6803 [12] or by our computational study. Thus the inhibition of urtA by $\mathrm{P}$ limitation is likely to be mediated by a different regulator other than SphR.

\section{Conclusion}

Different cyanobacterial species/ecotypes encode diverse sets of genes responsible for the utilization of various sources of phosphorus available in their environment. Unlike in E. coli, only a portion of cyanobacterial genes that are directly involved in phosphorus assimilation are under the regulation of SphR in some species/ecotypes. In the three genomes, i.e., CCMP1375, CC9902 and CC9311, where the SphR gene is missing, the Pho boxes have degraded to a level that is indistinguishable from the randomly occurring ones (Figure 2). Thus, a regulator seems to play an important role in retaining its binding sites. In addition, we have also shown that the predicted Pho regulons in cyanobacteria might include genes known to play important roles in other biological process, such as photosynthesis, carbon fixation, nitrogen assimilation, signal transduction and transcription regulation. These genes might serve as bridging points to couple the phosphorus assimilation pathway to the pathways underlying these biological processes.

\section{Methods \\ I. Materials}

The sequence and annotation files for the 19 sequenced cyanobacteria genomes were downloaded from the GenBankhttp://. These cyanobacterial genomes are Anabaena variabilis ATCC 29413 (ATCC29413), Cyanobacteria bacterium Yellowstone A Prime (Aprime), Cyanobacteria bacterium Yellowstone B Prime (Bprime), Gloeobacter violaceus PCC 7421 (PCC7421), Nostoc sp. PCC 7120 (采C7120), Prochlorococcus marinus CCMP1375 (ㄷMP1375), Prochlorococcus marinus MED4 (MED4), Prochlorococcus marinus MIT 9312 (MIT9312), Prochlorococcus marinus MIT9313 (MIT9313), Prochlorococcus marinus NATL2A (NATL2A), Synechococcus elongatus PCC 7942 (ㄷC7942), Synechococcus elongatus PCC 6301 (PCC6301), Synechococcus sp. WH8102 (WH8102), Synechococcus sp. CC9605 (CC9605), Synechococcus sp. CC9902 (ㄷ9902), Synechococcus sp. CC9311( (CC9311), Synechocystis sp. PCC 6803 (PCC6803), Thermosynechococcus elongates BF-1(BF-1) and Trichodesmium erythraeum IMS101 (IMS101).

\section{Prediction of operons}

Multi-gene operons were predicted using our JPOP program $[27,28]$ for each genome. For genes arranged in tandem on the same strand and not predicted to be part of an operon by JPOP, we considered them to form an operon if their intergenic distances are shorter than 45 base pairs. Genes that were not covered by this procedure were considered to each form a singleton operon.

\section{Prediction of orthologs}

We used the reciprocal best hit method [29] to predict orthologous genes between each two genomes by the 
BLASTP program with an E-value cutoff $10^{-6}$ for both directions.

\section{Phylogenetic footprinting}

Since the operons sphX-pstS1-C1-A1-B1-B1', pstS2-C2-A2B2, and phoA-nucH in PCC6803 are known to be regulated by SphR through at least three tandem Pho boxes in their promoter regions, we identified the orthologues (if exist) of these genes in the other 18 genomes. We pooled the entire upstream regions (if a region is longer than 800 bases, only the immediate upstream 800 bases were used) of these orthologues in each genome according to the predicted operons. If an intergenic region is shorter than 100 bases, then 10 bases in its upstream coding region was included. Then a motif containing two 8 bp tandem repeats with a linker of $3 \mathrm{bp}$ were searched in these sequences using the CUBIC program [15]. The identified motifs with a score above a pre-selected cutoff $(\mathrm{p}<0.001)$ were returned. A profile was built for these identified two tandem sequences plus the linkers. A second profile was constructed by combining the two conserved 8 pb blocks in all these sequences.

\section{Genome wide prediction of SphR binding sites}

The two profiles of Pho boxes constructed above were used to scan the genome sequences for all possible SphR binding sites using an algorithm that we developed previously [13], which is briefly described as follows with some modifications.

For each predicted transcriptional unit $U\left(g_{1}, \ldots, g_{n}\right)$ containing genes $g_{1}, \ldots, g_{n}$ in genome $G$, we extract the entire upstream intergenic region (if the region is longer than 800 bases, then only the immediate upstream 800 bases were extracted), denoted $I_{U\left(g_{1}, \ldots, g_{n}\right)}$ for scanning for possible binding sites. If an intergenic region is shorter than 100 bases, then 10 bases in its upstream coding region was included. All the extracted $I_{U\left(g_{1}, \ldots, g_{n}\right)}$ 's in the genome are denoted as set $I_{U}$. We also extracted a randomly chosen coding sequence with the same length as $I_{U\left(g_{1}, \ldots, g_{n}\right)}$ from $G$, denoted as $C_{U\left(g_{1}, \ldots, g_{n}\right)}$ for scanning for randomly occurring motifs. All the extracted $C_{U\left(g_{1}, \ldots, g_{n}\right)}$ 's in the genome are denoted as set $C_{U}$. We say that $I_{U\left(g_{1}, \ldots, g_{n}\right)}$ and $C_{U\left(g_{1}, \ldots, g_{n}\right)}$ are associated with $U\left(g_{1}, \ldots, g_{n}\right)$ and with each of the genes $g_{1}, \ldots$, and $g_{n}$ as well. The score of a putative binding site found in an extracted sequence $t$ ( $t \in I_{U}$ or $t \in$ $C_{U}$ ) by scanning with a profile $M$ is defined as

$$
\begin{gathered}
s_{M}(t)=\max _{h \subset t} \sum_{i=1}^{l} I_{i} \ln \frac{p(i, h(i))}{q(h(i))}, \\
I_{i}=\left(\sum_{b \in\{A, C, G, T\}} p(i, b) \ln \frac{p(i, b)}{q(b)}\right) / a, \\
a=\frac{n+1}{n+4} \ln (n+1)-\ln (n+4)-\frac{1}{n+4} \sum_{b \in\{A, C, G, T\}} \ln q(b)-\frac{n}{n+4} \ln _{b \in\{A, C, G, T\}} q(b),
\end{gathered}
$$

where $l$ is the length of the binding sites of $M, h$ any substring of $t$ with length $l, h(i)$ the base at position $i$ of $h$, $p(i, b)$ the relative frequency of base $b$ at position $i$ in $M$, $q(b)$ the relative frequency of base $b$ occurring in the background, and $n$ the number of sequences in $M$. A pseudocount 1 is added to the frequency of each base at each position in the profile when computing $p(i, b)$. The coefficient $a$ is used for normalization so that $I_{i}$ is in the region $[0,1]$.

When multiple profiles $M_{1}, \ldots, M_{z}$ are used for scanning, the score of concurrence of multiple putative binding sites in the sequence $t$ is defined as

$$
s_{M_{1} \ldots M_{z}}(t)=\sum_{j=1}^{z} s_{M_{j}}(t)
$$

We now define a score for concurrence of multiple binding sites in a sequence associated with a transcription unit by also considering the presence of similar motifs in the sequences associated with its orthologous genes in other genomes. Let $t$ be the extracted sequence $\left(t \in I_{U}\right.$ or $\left.t \in C_{U}\right)$ associated with a transcription unit $U\left(g_{1} \ldots g_{n}\right)$ in genome $T$. If $g_{i}$ has orthologues in $m_{i}$ closely related genomes $G_{1}, \ldots, G_{m i}$ let $o_{k}\left(g_{i}\right)$ be the same type of extracted sequence $\left(o_{k}\left(g_{i}\right) \in I_{U}\right.$ or $\left.o_{k}\left(g_{i}\right) \in C_{U}\right)$ associated with the orthologue of $g_{i}$ in genome $G_{k}$. Then the score of concurrence of the multiple binding sites in $t$ is redefined as

$$
s(t)=s_{M_{1} \ldots M_{z}}(t)+\max _{1<i<n} \sum_{j=1}^{z} \sum_{k=1}^{m_{i}} \frac{l_{j}-d_{i, j, k}}{m_{i} l_{j}} s_{M_{j}}\left(o_{k}\left(g_{i}\right)\right)
$$

where $d_{i, j, k}$, is the Hamming distance between the sequence found by using profile $M_{j}$ in $t$, and the corresponding sequence found in $o_{k}\left(g_{i}\right)$, and $l_{j}$ is the length of the sequences of profile $M_{j}$. If a group of genomes are highly similar to one another in their sequences, then the orthologues information among them will not be used for their respective binding site predictions to avoid counting non-functionally conserved sequences. In the present 
study, such groups are PCC6801 and PCC7942 as well as Aprime and Bprime.

When we consider all the extracted sequences in $I_{U}$ and $C_{U}$ in a genome, the scores of binding sites found in $v \in I_{U}$ and $w \in C_{U}, S_{I_{U}} \in s(v)$ and $S_{C_{U}} \in s(w)$, respectively, are random variables. Let $p\left(S_{I_{U}}>s\right)$ and $p\left(S_{C_{U}}>s\right)$ be the cumulative probability that $I_{U}$ and $C_{U}$ have putative binding sites with scores $s(v)>s$ and $s(w)>s$, respectively. To compute $p\left(S_{C_{U}}>s\right)$ for a genome, we generated 300 $C_{U\left(g_{1}, \ldots, g_{n}\right)}$ 's associated with each transcription unit $U\left(g_{1}, \ldots, g_{n}\right)$ in each genome and computed the score for each $C_{U\left(g_{1}, \ldots, g_{n}\right)}$ to avoid possible biased sampling. We then used the following log-odds ratio $(L O R)$ function to estimate the confidence of the predictions in a genome,

$$
L O R(s)=\ln \frac{p\left(S_{I_{U}}>s\right)}{p\left(S_{C_{U}}>s\right)} .
$$

Since $p\left(S_{C_{U}}>s\right)$ is the probability of type I error for testing the null hypothesis that $I_{U}$ does not contain a binding site when $S_{I_{U}}$ is greater than a cutoff $s_{c^{\prime}}$ we used it to estimate the false positive rate of our prediction, i.e., the $p$ value, and a cutoff 0.01 was used for the SphR binding site prediction in each genome.

\section{Abbreviations}

$\mathrm{P}_{\mathrm{i}^{\prime}}$ inorganic phosphate; LOR, log odds ratio; ATCC29413, Anabaena variabilis ATCC 29413; Aprime, Cyanobacteria bacterium Yellowstone A Prime; Bprime, Cyanobacteria bacterium Yellowstone B Prime; PCC7421, Gloeobacter violaceus PCC 7421; PCC7120, Nostoc sp. PCC 7120; CCMP1375, Prochlorococcus marinus CCMP1375; MED4, Prochlorococcus marinus MED4; MIT9312, Prochlorococcus marinus MIT 9312; MIT9313, Prochlorococcus marinus MIT9313; NATL2A, Prochlorococcus marinus NATL2A; PCC7942, Synechococcus elongatus PCC 7942; PCC6301, Synechococcus elongatus PCC 6301; WH8102, Synechococcus sp. WH8102; CC9605, Synechococcus sp. CC9605; CC9902, Synechococcus sp. CC9902; CC9311, Synechococcus sp. CC9311; PCC6803, Synechocystis sp. PCC 6803; BF-1, Thermosynechococcus elongates BF-1; and IMS101, Trichodesmium erythraeum IMS10.

\section{Authors' contributions}

ZS designed and conducted the experiments, and wrote the manuscript; VO designed and tested a scoring function, and implemented a part of the program; YX con- ceived the project and wrote the manuscript. All authors read and approved the final manuscript.

\section{Additional material}

\section{Additional file 1}

Predicted Pho boxes in 16 cyanobacterial genomes. Tables list the predicted SphR binding sites in 16 cyanobacterial genomes that encode the sphR gene.

Click here for file

[http://www.biomedcentral.com/content/supplementary/14712164-8-156-S1.pdf]

\section{Acknowledgements}

This research was supported in part by the US Department of Energy's Genomes to Life program [30] under project, "Carbon Sequestration in Synechococcus sp.: From Molecular Machines to Hierarchical Modeling" [3 I], National Science Foundation (NSF/DBI-035477I, NSF/ITR-IIS-0407204, NSF/DBI-0542 I 19, NSF/CCF-062 I 700) and by a start-up fund from the University of North Carolina at Charlotte to Z.C.S. We would like to thank Dr. Anthony Fodor for his critical reading of this manuscript and suggestions. We would also like to thank two anonymous reviewers for their suggestions which greatly improve this manuscript.

\section{References}

I. Whitton BA, Potts M: Introduction to the cyanobacteria. In Ecology of Cyanobacteria: their diversity in Time and Space Edited by: Whitton BA, Potts M. Kluwer Academic Publishers; 2003:I-II.

2. Field CB, Behrenfeld MJ, Randerson JT, Falkowski P: Primary production of the biosphere: integrating terrestrial and oceanic components. Science 1998, 28 I (5374):237-240.

3. Sanudo-Wilhelmy SA, Kustka AB, Gobler CJ, Hutchins DA, Yang M, Lwiza K, Burns J, Capone DG, Raven JA, Carpenter EJ: Phosphorus limitation of nitrogen fixation by Trichodesmium in the central Atlantic Ocean. Nature 200 I, 4 I I (6833):66-69.

4. Dyhrman ST, Chappell PD, Haley ST, Moffett JW, Orchard ED, Waterbury JB, Webb EA: Phosphonate utilization by the globally important marine diazotroph Trichodesmium. Nature 2006, 439(7072):68-7I.

5. Sundareshwar PV, Morris JT, Koepfler EK, Fornwalt B: Phosphorus limitation of coastal ecosystem processes. Science 2003, 299:563-565

6. Wu J, Sunda W, Boyle EA, Karl DM: Phosphate depletion in the western North Atlantic Ocean. Science 2000, 289(5480):759-762.

7. Thingstad TF, Krom MD, Mantoura RF, Flaten GA, Groom S, Herut B, Kress N, Law CS, Pasternak A, Pitta P, Psarra S, Rassoulzadegan F, Tanaka T, Tselepides A, Wassmann P, Woodward EM, Riser CW, Zodiatis G, Zohary T: Nature of phosphorus limitation in the ultraoligotrophic eastern Mediterranean. Science 2005, 309(5737): 1068-107|.

8. Wanner BL: Phosphorus assimilation and control of the phosphate regulon. In Escherichia coli and Salmonella: cellular and molecular biology Edited by: Neidhardt FC, Curtiss III R, INgraham JL, Lin ECC, Low KB, Magasanik B, Reznikoff WS, Riley M, Schaechter M, Umbarger HE. Washington D.C. , ASM Press ; 1996: I357-138I.

9. Hulett FM: The signal-transduction network for Pho regulation in Bacillus subtilis. Mol Microbiol 1996, 19(5):933-939.

10. Pragai Z, Allenby NE, O'Connor N, Dubrac S, Rapoport G, Msadek T, Harwood CR: Transcriptional regulation of the phoPR operon in Bacillus subtilis. J Bacteriol 2004, I 86(4): I | 82-। | 90.

II. Hirani TA, Suzuki I, Murata N, Hayashi H, Eaton-Rye Jj: Characterization of a two-component signal transduction system involved in the induction of alkaline phosphatase under phosphate-limiting conditions in Synechocystis sp. PCC 6803. Plant MolBiol 200I, 45: 133-144. 
12. Suzuki S, Ferjani A, Suzuki I, Murata N: The SphS-SphR two component system is the exclusive sensor for the induction of gene expression in response to phosphate limitation in synechocystis. J Biol Chem 2004, 279: I 3234-I 3240.

13. Su Z, Olman V, Mao F, Xu Y: Comparative genomics analysis of NtcA regulons in cyanobacteria: regulation of nitrogen assimilation and its coupling to photosynthesis. Nucleic Acid Res 2006, 33(16):5I56-5I7I.

14. Blanco AG, Sola M, Gomis-Ruth FX, Coll M: Tandem DNA recognition by PhoB, a two-component signal transduction transcriptional activator. Structure 2002, 10:701-713.

15. Olman $\mathrm{V}, \mathrm{Xu} \mathrm{D}, \mathrm{Xu} Y$ : CUBIC: identification of regulatory binding sites through data clustering. J Bioinform Comput Biol 2003, I(I):2I-40.

16. Scanlan DJ, West NJ: Molecular ecology of the marine cyanobacterial genera Prochlorococcus and Synechococcus. FEMS Microbiology Ecology 2002, 40 (I ): I- I2.

17. Martiny AC, Coleman ML, Chisholm SW: Phosphate acquisition genes in Prochlorococcus ecotypes: evidence for genomewide adaptation. Proc Natl Acad Sci U S A 2006, 103(33): I 2552-I 2557.

18. Jiang W, Metcalf WW, Lee KS, Wanner BL: Molecular cloning, mapping, and regulation of Pho regulon genes for phosphonate breakdown by the phosphonatase pathway of Salmonella typhimurium LT2. J Bacteriol 1995, I77:64 I I-642 I.

19. Kononova SV, Nesmeyanova MA: Phosphonates and their degradation by microorganisms. Biochemistry (Mosc) 2002, 67:184-195.

20. Dufresne A, Salanoubat M, Partensky F, Artiguenave F, Axmann IM, Barbe V, Duprat S, Galperin MY, Koonin EV, Le Gall F, Makarova KS, Ostrowski M, Oztas S, Robert C, Rogozin IB, Scanlan DJ, Tandeau de Marsac N, Weissenbach J, Wincker P, Wolf YI, Hess WR: Genome sequence of the cyanobacterium Prochlorococcus marinus SS I 20, a nearly minimal oxyphototrophic genome. Proc Natl Acad Sci U S A 2003, 100( I 7): 10020-10025.

21. Palenik B, Ren Q, Dupont CL, Myers GS, Heidelberg JF, Badger JH, Madupu R, Nelson WC, Brinkac LM, Dodson RJ, Durkin AS, Daugherty SC, Sullivan SA, Khouri H, Mohamoud Y, Halpin R, Paulsen IT: Genome sequence of Synechococcus CC93 I I: Insights into adaptation to a coastal environment. Proc Natl Acad Sci U S A 2006, I 03(36): I3555-13559.

22. Joint Genomics Institute [http://www.jgi.doe.gov/]

23. Rocap G, Larimer FW, Lamerdin J, Malfatti S, Chain P, Ahlgren NA, Arellano A, Coleman M, Hauser L, Hess WR, Johnson ZI, Land M, Lindell D, Post AF, Regala W, Shah M, Shaw SL, Steglich C, Sullivan MB Ting CS, Tolonen A, Webb EA, Zinser ER, Chisholm SW: Genome divergence in two Prochlorococcus ecotypes reflects oceanic niche differentiation. Nature 2003, 424(6952): $1042-1047$.

24. Moses AM, Chiang DY, Kellis M, Lander ES, Eisen MB: Position specific variation in the rate of evolution in transcription factor binding sites. BMC Evol Biol 2003, 3:19.

25. VanBogelen RA, Olson ER, Wanner BL, Neidhardt FC: Global analysis of proteins synthesized during phosphorus restriction in Escherichia coli. J Bacteriol 1996, 178:4344-4366.

26. Antelmann $\mathrm{H}$, Scharf $\mathrm{C}$, Hecker M: Phosphate starvation-inducible proteins of Bacillus subtilis: proteomics and transcriptional analysis. J Bacteriol 2000, I82:4478-4490.

27. Chen X, Su Z, Dam P, Palenik B, Xu Y, jiang T: Operon prediction by comparative genomics: an application to the Synechococcus sp. WH8 102 genome. Nucleic Acids Res 2004, 32:2 I 47-2 I 57.

28. Chen X, Su Z, Xu Y, Jiang T: Computational prediction of operons in Synechococcus sp. WH8 I 02. Genome Inform Ser Workshop Genome Inform 2004, I 5(2):21 I-222.

29. Mushegian AR, Koonin EV: A minimal gene set for cellular life derived by comparison of complete bacterial genomes. Proc Natl Acad Sci U S A 1996, 93:10268-10273.

30. Genome to Life Project [http://genomicsgtl.energy.gov/]

31. Carbon Sequestration in Synechococcus sp.: From Molecular Machines to Hierarchical Modeling [http:// www.genomes2life.org/]

32. Weblogo server [http://weblogo.berkeley.edu/logo.cgi]
Publish with Bio Med Central and every scientist can read your work free of charge

"BioMed Central will be the most significant development for disseminating the results of biomedical research in our lifetime. "

Sir Paul Nurse, Cancer Research UK

Your research papers will be:

- available free of charge to the entire biomedical community

- peer reviewed and published immediately upon acceptance

- cited in PubMed and archived on PubMed Central

- yours - you keep the copyright

Submit your manuscript here:

http://www.biomedcentral.com/info/publishing_adv.asp
BioMedcentral 